\title{
Drug-Drug Interaction Assessment and Identification in the Primary Care Setting
}

\author{
John Peabodya, b, c, f, Maria Czarina Acelajado ${ }^{\mathrm{d}}$, Tim Robert ${ }^{\mathrm{e}}$, Cheryl Hild ${ }^{\mathrm{e}}$, \\ Joshua Schrecker ${ }^{\mathrm{e}}$, David Paculdo ${ }^{\mathrm{c}}$, Mary Tran ${ }^{\mathrm{c}}$, Elaine Jeter ${ }^{\mathrm{e}}$
}

\begin{abstract}
Background: Drug-drug interactions (DDIs) are ubiquitous, harmful and a leading cause of morbidity and mortality. With an aging population, growth in polypharmacy, widespread use of supplements, and the rising opioid abuse epidemic, primary care physicians (PCPs) are increasingly challenged with identifying and preventing DDIs. We set out to evaluate current clinical practices related to identifying and treating DDIs and to determine if opportunities to increase prevention of DDIs and their adverse events could be identified.
\end{abstract}

Methods: In a nationally representative sample of 330 board-certified family and internal medicine practitioners, we evaluated whether PCPs assessed DDIs in the care they provided for three simulated patients. The patients were taking common prescription medications (e.g. opioids and psychiatric medications) along with other common ingestants (e.g. supplements and food) and presented with symptoms of DDIs. Physicians were scored on their ability to inquire about the patient's medications, investigate possible DDIs, evaluate the patient, and provide treatment recommendations. We scored the physicians' care recommendations against evidence-based criteria, including overall care quality and treatment for DDIs.

Results: Average overall quality of care score was $50.5 \% \pm 12.0 \%$. Despite $>99 \%$ self-reported use of medication reconciliation practices and tools, physicians identified DDIs in only $15.3 \%$ of patients, with $15.5 \% \pm 20.3 \%$ of DDI-specific treatment by the physicians.

Conclusions: PCPs in this study did not recognize or adequately treat DDIs. Better methods are needed to screen for DDIs in the primary care setting.

Keywords: Drug-drug interaction; Drug-food interaction; Drug-supplement interaction; Medication reconciliation; Adverse drug event;

Manuscript submitted July 31, 2018, accepted August 31, 2018

aUniversity of California, San Francisco, CA, USA

bUniversity of California, Los Angeles, CA, USA

${ }^{\mathrm{C}} \mathrm{QURE}$ Healthcare, San Francisco, CA, USA

dAthens Limestone Hospital, Athens, AL, USA

${ }^{\mathrm{e}}$ Aegis Sciences Corporation, Nashville, TN, USA

${ }^{\mathrm{f} C}$ Corresponding Author: John Peabody, Suite 200, 450 Pacific Ave., San Fran-

cisco, CA 94133, USA. Email: jpeabody@qurehealthcare.com

doi: https://doi.org/10.14740/jocmr3557w
Primary care; Psychiatric medications; Opioids

\section{Introduction}

Drug-drug interactions (DDIs) are ubiquitous, costly and a leading cause of morbidity and mortality worldwide [1]. In the United States alone, DDIs contribute to $20 \%$ of all adverse drug events [2], which cause nearly 770,000 deaths and result in $\$ 30$ billion [3] to $\$ 180$ billion in healthcare expenditures [4] and four hospitalizations per 1,000 people annually [5].

An aging population, polypharmacy treatment, supplement use and drug abuse will only make this problem worse. Today the average patient over 65 is on four medications [6] and, by $2020,18 \%$ of the US population will be over 65 [7]. Polypharmacy, defined as concomitant use of five or more medications, is associated with an $80 \%$ risk of experiencing a DDI [8]. Today, in the US, approximately $36 \%$ of adults can be categorized as polypharmacy patients [9]. The risk of potentially life-threatening drug interactions extends beyond just prescription medications as well. Dietary and herbal supplement use is both common and rapidly rising throughout the US [10]. Concurrent use of supplements with just one prescription medication is associated with a 1 in 25 risk of a DDI [11]. Certain foods and drinks may interact with medications as well and these drug-food interactions also go unrecognized.

High quality, safe care requires that DDIs be recognized and avoided. Existing tools to evaluate the risk of DDIs, which include physician inquiry, automated drug interaction checking software and pharmacy medication reconciliation reports, can help but so far have failed to increase physician awareness to the problem of DDIs [12]. There may be several reasons for this, all related to physician awareness and inadequate data. The patient medication records on which all tools rely are often incomplete or they may not include records from all providers of a patient. Additionally, patients may unintentionally or intentionally omit describing medications to their provider. In particular, the misuse of prescription medications has risen steadily over the past decade [13], with opioids and central nervous system (CNS) depressants being among the most common classes of drugs misused [14]. Opioids, CNS depressants, and antibiotics are also among the most commonly prescribed medications in the US, only adding to the problem [15]. What is often missed is that these agents, particularly narcotics, have 
the potential to cause significant harm when they interact with other prescription drugs, non-prescription drugs and food supplements.

DDIs are unique in that they are iatrogenic and almost entirely preventable. However, little is known about how well this issue is recognized, understood and treated, particularly among front-line primary care practitioners (PCPs). We set out to evaluate current care practices related to the identification and treatment of DDIs and to determine if we could identify opportunities to increase prevention of DDIs and their adverse events.

\section{Materials and Methods}

We conducted a prospective, cross-sectional study of DDI preventive care practices among PCPs practicing in the US from May to July 2018. We evaluated the DDI screening, workup, and care recommendations of board-certified family and internal medicine physicians as they cared for identical simulated patients known as Clinical Performance and Value $\left(\mathrm{CPV}^{\circledR}\right)$ vignettes.

\section{Ethics}

This study was conducted in accordance with ethical standards, approved by the Advarra Institutional Review Board, Columbia, MD, and listed in clinicaltrials.gov (NCT03581994). Informed consent was obtained from all participants.

\section{Physician selection}

We recruited participants from a nationally representative list of over 25,000 PCPs. The recruitment lists were compiled from relevant contact resources, including medical association workforce databases and list serves, hospital organization physician rosters, and national medical conference attendees. Between May and July 2018, we invited randomly selected physicians from the compiled list. Participants were screened for eligibility using a 20-question physician questionnaire. Physicians who met the eligibility criteria were invited to participate until the study sample size of 330 physicians was achieved.

Eligible participants had to: 1) be physicians either boardcertified in internal medicine or family medicine; 2 ) practice in a non-academic setting; 3 ) have between 2 and 30 years of post-residency practice, and 4) have an active panel of over 500 patients with an adult patient load of more than 50\%. Sampling was stratified so that physician characteristics including regional geography, age, gender, and practice size were representative of the PCP population nationally (Supplementary Table 1) (www.jocmr.org).

\section{Clinical Performance and Value vignettes}

CPV simulated patients require physicians to care for patients as they would in their office setting [16]. The tool allows physicians to make inquiries of the patient, review histories, and order laboratory tests and procedures to recreate an actual patient visit. Open-ended queries in the CPVs are divided into five domains of care: 1) taking a history; 2) performing a physical; 3) ordering diagnostic workup; and 4) making a diagnosis with 5) treatment plan and follow-up. In each case there are between 49 and 72 evidence-based criteria evaluated. Scoring was done by two physicians, working independently, using explicit, pre-determined criteria with a third physician adjudicating in the case of a disagreement on any of the individual criteria. Each domain as well as the overall performance thus has a score of between $0 \%$ and $100 \%$. Because all physicians are caring for the same patients, CPV vignettes adjust for case-mix variation and provide a clear measurement of clinical practice variation.

\section{CPV patients}

We created nine CPV cases to be cared for by participating physicians. All cases presented participants with one of three commonly prescribed medication classes (opioids, CNS depressants, or antibiotics) in addition to common substances (over the counter medications (OTCs), supplements, food, alcohol, or other prescription medications) that may cause one or more DDIs. The cases are summarized in Supplementary Table 2 (www.jocmr.org).

\section{Analysis}

The primary outcome was to evaluate current DDI assessment practices and to identify barriers and opportunities in preventing DDIs in the primary care setting. More specifically, we sought to: 1) determine the frequency in which PCPs were able to identify, diagnose, and treat DDIs in simulated CPV patients and 2) evaluate impact of provider characteristics (e.g., age, gender, practice setting) and clinical practice characteristics (e.g., asking about history of medications, ordering a presumptive or definitive drug test) on the likelihood of diagnosis and treatment of DDIs. Chi-squared tests and logistic regression modeling were used for analyses involving binary outcome variables (e.g., diagnosing a DDI), and $t$-tests and linear regression modeling were used for analysis of continuous outcomes (e.g., diagnosis-treatment score). All analyses were conducted in Stata 14.2.

\section{Results}

\section{Physician-practice characteristics}

A total of 330 board-certified physicians met the eligibility criteria and completed the physician questionnaire and patient cases (Table 1). About $49.1 \%$ were board-certified in family medicine, $49.7 \%$ in internal medicine, and $1.2 \%$ in both. Males made up $76.7 \%$ of participants and females $23.3 \%$. Among the participants, $6.4 \%$ were under 40 years old, $60.9 \%$ were be- 
Table 1. Baseline Provider Characteristics, Self-Reported

\begin{tabular}{|c|c|}
\hline & $\mathbf{N}=\mathbf{3 3 0}$ \\
\hline Male & $76.7 \%$ \\
\hline \multicolumn{2}{|l|}{ Age } \\
\hline $40-55$ & $60.9 \%$ \\
\hline$>55$ & $32.7 \%$ \\
\hline Family medicine & $49.1 \%$ \\
\hline Internal medicine & $49.7 \%$ \\
\hline Both & $1.2 \%$ \\
\hline Fellowship & $14.6 \%$ \\
\hline Years in practice & $20.1 \pm 6.9$ \\
\hline South & $30.9 \%$ \\
\hline West & $20.9 \%$ \\
\hline \multicolumn{2}{|l|}{ Locale } \\
\hline Urban & $26.7 \%$ \\
\hline Suburban & $60.9 \%$ \\
\hline Rural & $12.4 \%$ \\
\hline \multicolumn{2}{|l|}{ Practice type } \\
\hline Solo private & $23.3 \%$ \\
\hline Accountable care organization & $24.2 \%$ \\
\hline Solo practice & $23.3 \%$ \\
\hline Group practice & $66.4 \%$ \\
\hline Hospital-based & $8.2 \%$ \\
\hline Integrated delivery system & $11.5 \%$ \\
\hline Network model HMO & $1.2 \%$ \\
\hline Staff-model HMO (employed) & $1.2 \%$ \\
\hline Staff-model HMO (FFS or group, contracted) & $1.2 \%$ \\
\hline Other & $1.8 \%$ \\
\hline \multicolumn{2}{|c|}{ Type of medication reconciliation used (physicians were able to choose $>1$ ) } \\
\hline Pharmacy/medication reconciliation & $88.2 \%$ \\
\hline Presumptive drug tests & $57.3 \%$ \\
\hline Definitive drug tests & $37.3 \%$ \\
\hline Digital pills & $0.0 \%$ \\
\hline Self-report & $62.7 \%$ \\
\hline
\end{tabular}


Table 1. Baseline Provider Characteristics, Self-Reported - (continued)

\begin{tabular}{|c|c|}
\hline & $\mathbf{N}=\mathbf{3 3 0}$ \\
\hline EMR/automated software & $74.6 \%$ \\
\hline None & $1.0 \%$ \\
\hline$\leq 12$ months & $41.5 \%$ \\
\hline 13 - 24 months & $17.0 \%$ \\
\hline Never taken & $20.9 \%$ \\
\hline Receive quality bonus & $59.7 \%$ \\
\hline If yes, metrics for preventive measures & $88.8 \%$ \\
\hline Active panel size & $2,542 \pm 1,526$ \\
\hline Consume $\geq 2$ alcoholic beverages/month & $55.4 \%$ \\
\hline \multicolumn{2}{|l|}{ Payer type } \\
\hline Medicare & $34.3 \%$ \\
\hline Medicaid & $10.8 \%$ \\
\hline Commercial & $48.1 \%$ \\
\hline Self & $5.4 \%$ \\
\hline Other & $1.4 \%$ \\
\hline
\end{tabular}

tween 40 and 55 years, and $32.7 \%$ were over 55 years. On average, these participants had $20.1 \pm 6.9$ years of practice experience and worked in a private, non-hospital-based practice $(93.9 \%)$ in an urban/suburban location (87.6\%). The average active patient panel size of participant physicians was just over 2,500 patients. Overall, participants estimated that in their patient panel $41.2 \%$ were on five or more medications, $55.4 \%$ consumed at least two alcoholic beverages per month, and $13.9 \%$ were on opioid analgesics.

From the survey, we learned that nearly every physician participant (99\%) felt they reconciled medications and regularly monitored their patients for potential DDIs. The most common methods were pharmacy medication reconciliation (PMR) reports $(88.2 \%)$, EMR or other automated software alerts $(74.6 \%)$ and patient self-reported histories (62.7\%). Additionally, $79.1 \%$ of providers indicated that within the past 3 years they had received CME in pain management, and more than half $(59.7 \%)$ reported that they had received a quality bonus in the past which included metrics for preventive measures $(88.8 \%)$.

\section{Variability of provider practice}

Overall, the 330 physician participants cared for a total of 990 simulated patients with each individual participant taking care of three CPV patients. The cases were scored on the ability of the physicians to identify, workup, diagnose and treat these patients presenting with a medical complaint, and signs and symptoms of a potential DDI.

We observed a high degree of practice variation among the participants (Fig. 1). The average overall quality of care score for all participants was $50.5 \% \pm 12.0 \%$ out of a total potential score of $100 \%$. Across care domains, the highest average score was achieved in physical examination $(79.2 \% \pm 24.8 \%)$ and the lowest in diagnosis-treatment $(22.9 \% \pm 16.9 \%)$ (Table 2).

In just over half the cases $(55.1 \%)$, physicians correctly

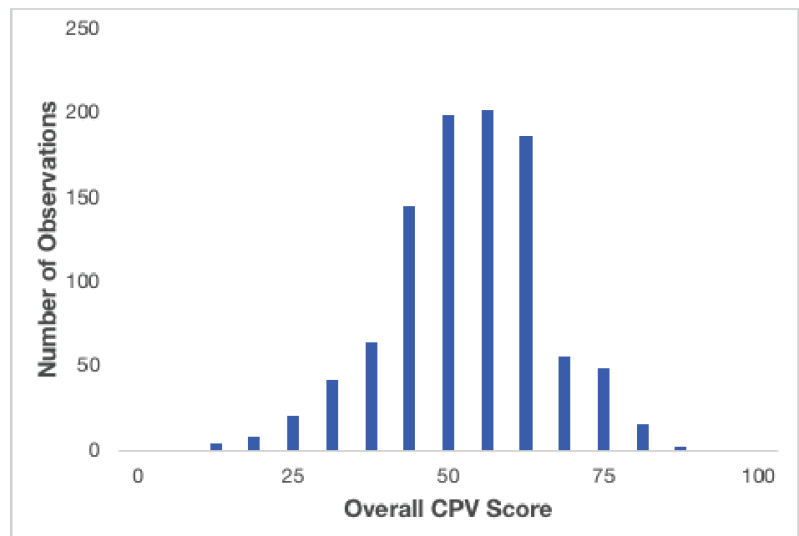

Figure 1. Histogram of overall CPV scores. 
Table 2. CPV Results

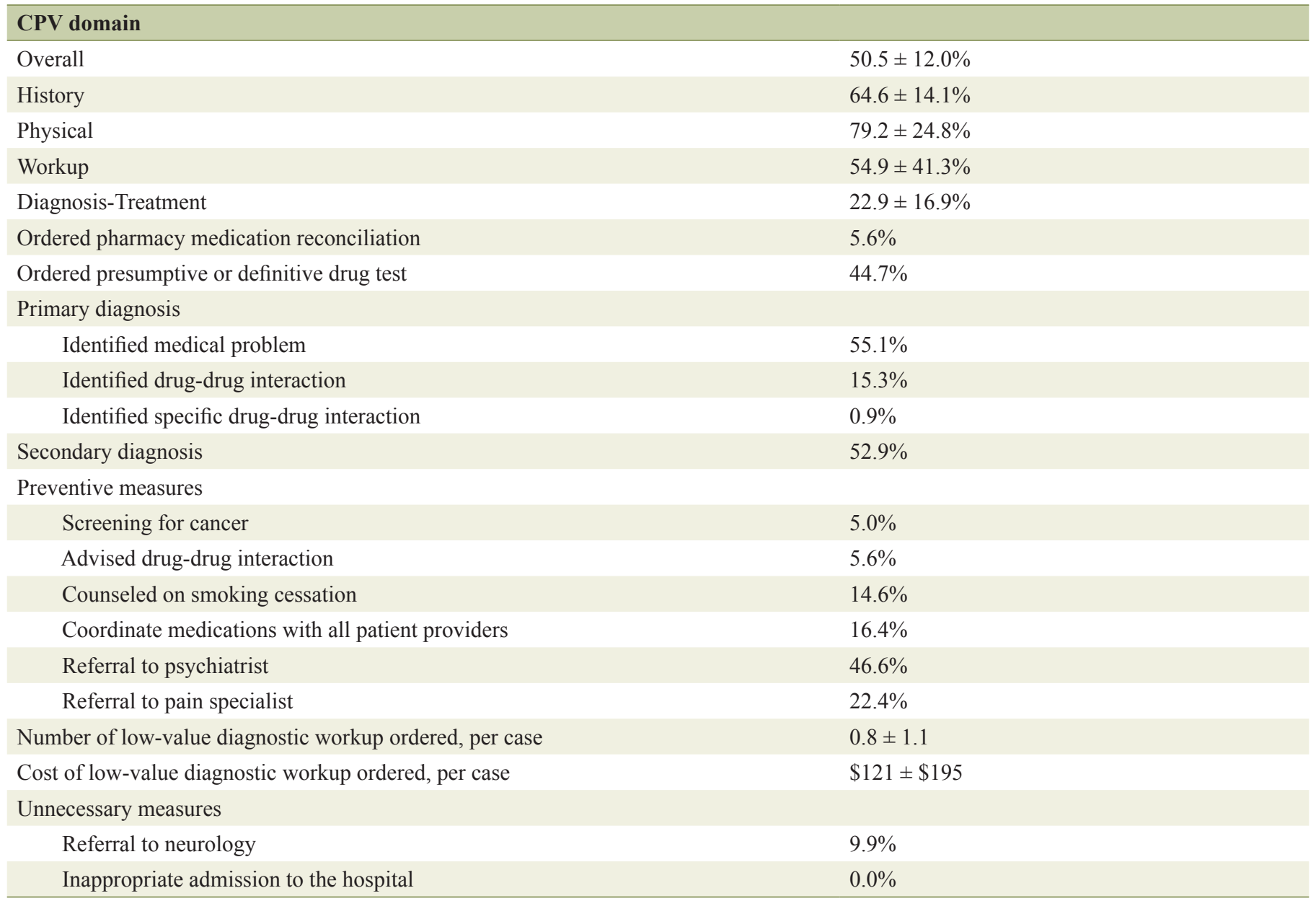

diagnosed their patients' primary medical problem, such as depression, palpitations or increased somnolence. However, they were only able to identify the underlying DDI causing the medical problem $15.3 \%$ of the time and the specific DDI even less often than that $(0.9 \%)$. Also, while nearly nine out of 10 physicians self-reported regular use of PMR in their practice, PMR reports were ordered only in $5.6 \%$ of the cases to look for a DDI. Those few who did order a PMR were, however, $42 \%$ more likely to identify the DDI diagnosis (95\% CI: $-28 \%$ $-182 \%)$.

Looking specifically at DDI evaluation and what was ordered to help determine patient diagnosis, physicians ordered a presumptive drug test (e.g. qualitative immunoassay and point of care testing) alone in $32.4 \%$ of cases; in $7.0 \%$ of cases physicians instead ordered a definitive drug test that used specific and sensitive analytical methods (e.g. liquid chromatography/ mass spectrometry) to confirm if a drug was present and, if so, at what concentrations. Additionally, physicians ordered both a presumptive drug test and then a definitive drug test $5.4 \%$ of the time. Those who ordered the presumptive drug test were marginally more likely $(6 \%)$ to make the diagnosis of a DDI (95\% CI: $-28 \%$ - 56\%) compared to those that did not order either drug test. In contrast, those who ordered a definitive drug test were 53\% more likely to make the DDI diagnosis (95\% CI, -19\% - 189\%). We observed a similar percentage increase for those who ordered both tests $(57.5 \%$; $95 \% \mathrm{CI}$ : $-22.2 \%-219.0 \%$ ), although these increases failed to achieve significance.

There were other indicators that recognition of DDI was wanting. Physicians indicated they would coordinate medications with other clinical providers only $16.4 \%$ of the time and only $5.6 \%$ indicated they would counsel their patients directly on the risks and issues of DDIs. Those that identified and diagnosed the underlying DDI, however, offered DDI counselling $32.7 \%$ of the time, while those that missed the DDI diagnosis failed to do so at all $(0.0 \%, \mathrm{P}<0.001)$.

\section{Physician and patient characteristics, regression modeling}

To determine opportunities to prevent DDIs and their adverse events, we performed linear and logistic regression analyses. The models assessed for associations between physician and patient characteristics to the overall score, diagnosis-treatment accuracy, and identification of a DDI or specification of DDIrelated treatments. 
Table 3. Logistic Regression to Identify a Potentially Harmful DDI

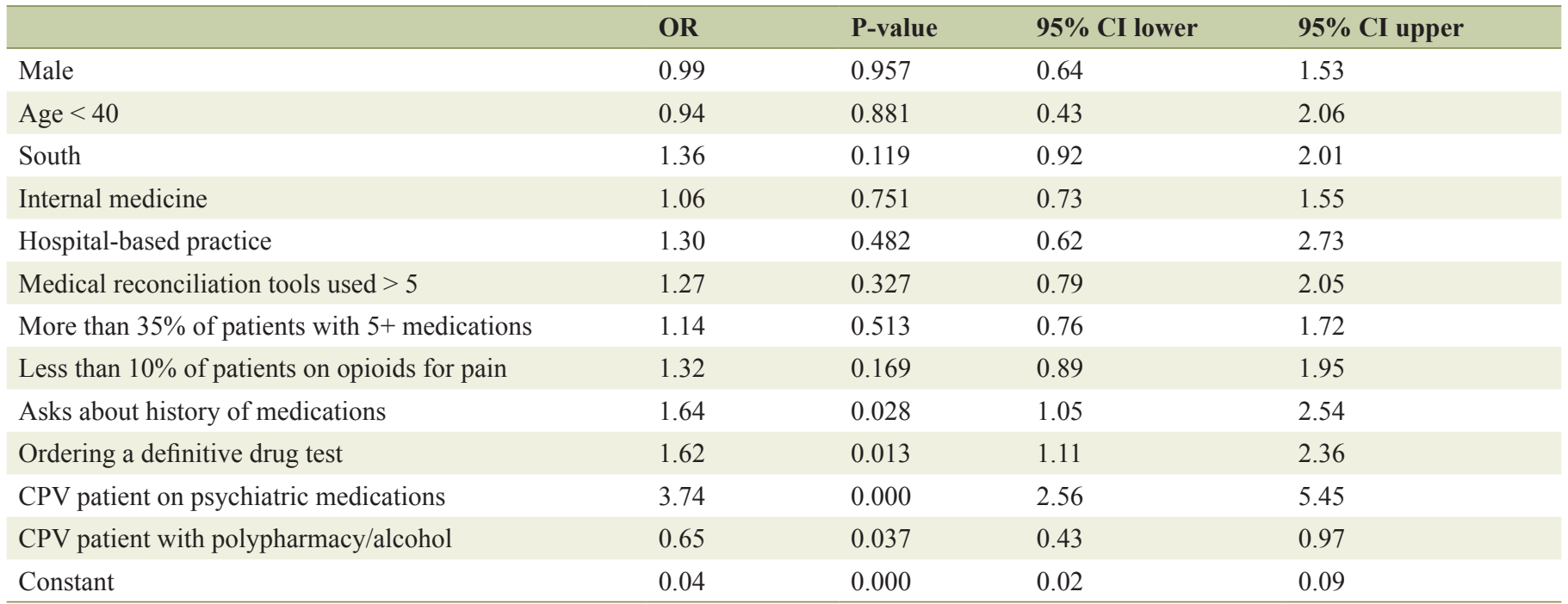

Several physician characteristics predicted overall quality of care score. Women $(\mathrm{P}=0.066)$ and physicians under the age of $55(\mathrm{P}=0.001)$ scored $1.7 \%$ and $5.4 \%$, respectively, higher than their counterparts. Physicians in the South or West scored $3.3 \%$ more than their counterparts in the Northeast and Midwest $(\mathrm{P}<0.001)$. When we restricted the regression to only the diagnosis-treatment domain, which includes all care (both DDI related and not), these differences remained significant.

We found that caring for a patient on psychiatric medications (OR: 3.74, 95\% CI: 2.56 - 5.45) suggested more to physicians that they should look for a DDI. This was not the case for polypharmacy or high alcohol consuming patients who were not as likely to be diagnosed with a DDI (OR: $0.65,95 \% \mathrm{CI}$ : 0.43 - 0.97) (Table 3). When physicians ordered a definitive drug test, this was linked to making a DDI diagnosis (OR: 1.62, 95\% CI: 1.11 - 2.36).

We investigated how providers did in the treatment of DDIs patients; they did poorly overall, providing only $15.5 \%$ $\pm 20.3 \%$ of DDI-related treatment and $21.5 \% \pm 26.2 \%$ of non-DDI-related treatment. Those who identified the DDI performed more than twice as much DDI-related treatment than those who did not $(28.8 \% \pm 24.0$ vs. $13.2 \% \pm 18.6 \%, \mathrm{P}$ $<0.001$ ), although their results were still low. Among specific DDI-related treatment items, in addition to being more likely to provide DDI counseling (as noted previously), those who identified and diagnosed the DDI identified the interacting drug or supplement (medication, antibiotic, supplement, etc.) at a significantly higher rate $(45.3 \%$ vs. $15.0 \%, \mathrm{P}<0.001)$ compared to those who did not.

Notwithstanding the poor overall treatment for DDIs, $41.6 \%$ of physicians provided "some" DDI-related treatment; that is, they performed at least one DDI-related treatment item for their patients. In logistic regression, those who identified the DDI were significantly more likely to provide some form of DDI-related treatment (OR: 3.32, 95\% CI: 2.21 - 4.99) (Table 4). We broke the results out by those who recognized and diagnosed a DDI versus those who missed the diagnosis. Us- ing a Chi-squared analysis, we found some DDI-related treatment was performed in $69.5 \%$ versus $36.6 \%$ of the patients, respectively $(\mathrm{P}<0.001)$.

Doctors who were hospital-based were more likely to treat a DDI (OR: 2.59, 95\% CI: 1.41 - 4.76). They were also more likely to provide better treatment if they ordered a definitive drug test (OR: $1.51,95 \%$ CI: 1.13 - 2.02) or had a patient on a psychiatric medication (OR: $1.86,95 \% \mathrm{CI}: 1.36-2.53$ ), but patients having polypharmacy or alcohol consumption issues (OR: $0.25,95 \%$ CI: $0.18-0.34$ ) were associated with less DDI-specific treatment.

\section{Discussion}

Despite the fact that drug-drug interactions are almost entirely avoidable there has been a dearth of investigations and few insights on how to improve patient care [17]. With an aging demographic, the widespread use of opiates and supplements, and the growth of polypharmacy, the problem of DDIs will only worsen in the coming decade. Despite these dangerous clinical consequences and enormous economic impact, it appears that physicians may not recognize and treat DDIs [18]. To better understand the clinical practice dimensions of the DDI problem, we conducted a large study among a nationally representative sample of 330 primary care practitioners caring for common types of patients with DDIs.

We found that physicians recognized DDIs in only $15.3 \%$ of 990 cases in this study. Our findings align with what others have found in the literature but suggest that the problem is far worse than these previous estimates [12, 18, 19]. Our more worrisome estimates may be more accurate because we used simulated patients wherein the patient's diagnosis is known, and we had complete data on every participant in the study: two problems that have plagued previous studies.

There is ample evidence that CPV simulations capture gaps in care quality and accurately detect practice variation 
Table 4. Performing Any DDI-related Treatment

\begin{tabular}{|c|c|c|c|c|}
\hline & OR & P-value & 95\% CI lower & 95\% CI upper \\
\hline Male & 0.73 & 0.066 & 0.52 & 1.02 \\
\hline Age $<40$ & 0.95 & 0.859 & 0.52 & 1.71 \\
\hline Internal medicine & 0.86 & 0.324 & 0.65 & 1.15 \\
\hline Hospital-based practice & 2.59 & 0.002 & 1.41 & 4.76 \\
\hline More than $35 \%$ of patients with $5+$ medications & 0.99 & 0.953 & 0.73 & 1.35 \\
\hline Less than $10 \%$ of patients on opioids for pain & 1.12 & 0.462 & 0.83 & 1.51 \\
\hline Asks about history of medications & 1.04 & 0.821 & 0.76 & 1.42 \\
\hline Ordering a definitive drug test & 1.51 & 0.006 & 1.13 & 2.02 \\
\hline Identified a drug-drug interaction exists & 3.32 & 0.000 & 2.21 & 4.99 \\
\hline
\end{tabular}

broadly similar to the results that we found in this study [20]. We identified several gaps in care, including a reliance on inappropriate presumptive drug testing for DDIs. Positive presumptive drug tests did not translate into significant increases in DDI-related diagnostic or therapeutic accuracy. Equally worrisome, even after physicians identified a potential DDI, they did not discuss the causes and effects of drug interactions with their patients. Other studies have similarly found that physicians educate their patients on drug-drug, drug-food, and adverse events less than $25 \%$ of the time [21]. Perhaps most significantly, while hospital-based providers, women, and younger physicians performed slightly better than the average primary care physician, these results show that the problem of recognizing and treating DDIs is ubiquitous.

The widespread failure to recognize DDIs was mitigated in a limited number of ways. Those that ordered a PMR were $19 \%$ more likely than those that ordered a presumptive drug test to recognize a DDI. Routine presumptive testing, however, was of only marginal help in making the diagnosis or providing better treatment. Those that ordered a definitive drug test were $23 \%$ more likely to diagnose and provide appropriate DDI care compared to those that ordered a PMR. While it appears that the availability of definitive drug test results improved provider's ability to identify DDIs, it should be noted that the majority of drugs and substances that cause interactions are not part of routine definitive test offerings. Thus, reliance on a definitive drug test that captures relatively few substances capable of interacting with other prescribed medications would likely leave gaps in appropriate identification of DDIs.

It is worth noting that virtually every family practitioner and internist in this study felt that they reconciled medications and regularly monitored their patients for potential DDIs by using a combination of PMR reports, software alerts and taking a careful history. Moreover four out of five participants had done related CME in the past 3 years and more than half were incentivized on the quality of care they provided. Discrepan- cies between reported and actual testing, as well as between testing and correct DDI identification and care suggest much more must be done to incorporate more effective DDI recognition and prevention in routine care.

Increasing DDI awareness will not be easy. This study shows that the problem is widespread and occurs across a range of patients including those using opioids, other commonly prescribed medications and over the counter products. Gaps in DDI care also extend across the country and are found amongst both family practitioners and general internists. Educational outreach is one possible solution, although the fact that $79.1 \%$ of the participants in this study had recently undergone CME in pain management dampens enthusiasm. Software tools have been shown to help [22] as have team-based approaches involving pharmacists [23]. More of these solutions could be implemented. Other system-level interventions, such as mandating medication reconciliation and EMR interventions are another approach. This study also clearly shows that current diagnostic tests for DDIs are underwhelming and better technology and testing will be critical to recognizing and treating DDIs. In the future, reliance on simple presumptive drug testing will have to give way to more comprehensive, definitive drug testing for DDIs when interactions are potentially present.

Although efforts were made to match demographics of practicing PCPs in the US, we had a higher representation of men and middle age physicians in our final participant population. The participating physicians reported having somewhat fewer patients who consumed alcohol $(55.8 \%$ vs. $73 \%)$ or were on opioid analgesics (13.9\% vs. 38\%) than what was expected in the general population, although the underreporting of opioid prescribing has been reported elsewhere [24]. We studied DDIs ranging from antibiotics to analgesics to CNS depressants with alcohol and opioids, but there is a legion of equally serious other DDIs, such as statin interactions, that exist and are likely even more underrecognized than the ones included 
in this study. This study also only examined PCP identification of potential DDIs and did not delve into how specialists would respond to these same CPV cases, which could be explored with simulations. Other considerations, such as patients with renal impairment or even a genetic polymorphism affecting drug metabolism, would also be of interest and readily studied using CPVs. For now, these gaps are the subject of future studies that can flesh out the impact of other interactions, such as those proposed above, and determine other DDIs that should be pursued as investigators assess the scope and scale of this problem.

\section{Declarations}

QURE, LLC, whose intellectual property was used to prepare the cases and collect the data, was contracted by Aegis Sciences Corporation. Otherwise, no disclosure to report.

\section{Funding Support}

This study was funded by Aegis Sciences Corporation, Nashville, Tennessee.

\section{References}

1. Lepakhin VK. Safety of Medicines: A guide to detecting and reporting adverse drug reactions. 2002. World Health Organization: Geneva, Switzerland. Available at: http://apps.who.int/iris/bitstream/10665/67378/1/WHO_ EDM_QSM_2002.2.pdf.

2. Magro L, Moretti U, Leone R. Epidemiology and characteristics of adverse drug reactions caused by drug-drug interactions. Expert Opin Drug Saf. 2012;11(1):83-94.

3. Sultana J, Cutroneo P, Trifiro G. Clinical and economic burden of adverse drug reactions. J Pharmacol Pharmacother. 2013;4(Suppl 1):S73-77.

4. Ernst FR, Grizzle AJ. Drug-related morbidity and mortality: updating the cost-of-illness model. J Am Pharm Assoc (Wash). 2001;41(2):192-199.

5. Shehab N, Lovegrove MC, Geller AI, Rose KO, Weidle NJ, Budnitz DS. US Emergency Department Visits for Outpatient Adverse Drug Events, 2013-2014. JAMA. 2016;316(20):2115-2125.

6. Charlesworth CJ, Smit E, Lee DS, Alramadhan F, Odden MC. Polypharmacy Among Adults Aged 65 Years and Older in the United States: 1988-2010. J Gerontol A Biol Sci Med Sci. 2015;70(8):989-995.

7. US Census Bureau. National Population Projections, 2017. Accessed June 2018. Available at: www.census. gov/programs-surveys/pproj.html.

8. Doan J, Zakrzewski-Jakubiak H, Roy J, Turgeon J, Tannenbaum C. Prevalence and risk of potential cytochrome P450-mediated drug-drug interactions in older hospitalized patients with polypharmacy. Ann Pharmacother. 2013;47(3):324-332.
9. Qato DM, Wilder J, Schumm LP, Gillet V, Alexander GC. Changes in Prescription and Over-the-Counter Medication and Dietary Supplement Use Among Older Adults in the United States, 2005 vs 2011. JAMA Intern Med. 2016;176(4):473-482.

10. Axon DR, Vanova J, Edel C, Slack M. Dietary Supplement Use, Knowledge, and Perceptions Among Student Pharmacists. Am J Pharm Educ. 2017;81(5):92.

11. Qato DM, Alexander GC, Conti RM, Johnson M, Schumm P, Lindau ST. Use of prescription and over-the-counter medications and dietary supplements among older adults in the United States. JAMA. 2008;300(24):2867-2878.

12. Tam VC, Knowles SR, Cornish PL, Fine N, Marchesano R, Etchells EE. Frequency, type and clinical importance of medication history errors at admission to hospital: a systematic review. CMAJ. 2005;173(5):510-515.

13. Blanco C, Alderson D, Ogburn E, Grant BF, Nunes EV, Hatzenbuehler ML, Hasin DS. Changes in the prevalence of non-medical prescription drug use and drug use disorders in the United States: 1991-1992 and 2001-2002. Drug Alcohol Depend. 2007;90(2-3):252-260.

14. SAMHSA. Substance abuse center for behavioral health statistics and quality. Results from the 2016 national survey on drug use and health: detailed tables. Published September 7, 2017. Accessed May 2018. Available at: https://www.samhsa.gov/data/sites/default/files/NSDUHDetTabs-2016/NSDUH-DetTabs-2016.htm.

15. Zhong W, Maradit-Kremers H, St Sauver JL, Yawn BP, Ebbert JO, Roger VL, Jacobson DJ, et al. Age and sex patterns of drug prescribing in a defined American population. Mayo Clin Proc. 2013;88(7):697-707.

16. Peabody JW, Luck J, Glassman P, Dresselhaus TR, Lee M. Comparison of vignettes, standardized patients, and chart abstraction: a prospective validation study of 3 methods for measuring quality. JAMA. 2000;283(13):1715-1722.

17. Indermitte J, Reber D, Beutler M, Bruppacher R, Hersberger KE. Prevalence and patient awareness of selected potential drug interactions with self-medication. J Clin Pharm Ther. 2007;32(2):149-159.

18. Hines LE, Malone DC, Murphy JE. Recommendations for generating, evaluating, and implementing drug-drug interaction evidence. Pharmacotherapy. 2012;32(4):304313.

19. Bucher HC, Achermann R, Stohler N, Meier CR. Surveillance of Physicians Causing Potential Drug-Drug Interactions in Ambulatory Care: A Pilot Study in Switzerland. PLoS One. 2016;11(1):e0147606.

20. Kubal T, Letson DG, Chiappori AA, Springett GM, Shimkhada R, Tamondong Lachica D, Peabody JW. Longitudinal cohort study to determine effectiveness of a novel simulated case and feedback system to improve clinical pathway adherence in breast, lung and GI cancers. BMJ Open. 2016;6(9):e012312.

21. Yi ZM, Zhi XJ, Yang L, Sun SS, Zhang Z, Sun ZM, Zhai SD. Identify practice gaps in medication education through surveys to patients and physicians. Patient Prefer Adherence. 2015;9:1423-1430.

22. Kheshti R, Aalipour M, Namazi S. A comparison of five common drug-drug interaction software programs re- 
garding accuracy and comprehensiveness. J Res Pharm Pract. 2016;5(4):257-263.

23. Lopez-Martin C, Garrido Siles M, Alcaide-Garcia J, Faus Felipe V. Role of clinical pharmacists to prevent drug interactions in cancer outpatients: a single-centre experi- ence. Int J Clin Pharm. 2014;36(6):1251-1259.

24. Adams NJ, Plane MB, Fleming MF, Mundt MP, Saunders LA, Stauffacher EA. Opioids and the treatment of chronic pain in a primary care sample. J Pain Symptom Manage. 2001;22(3):791-796. 